\title{
Enhancement of Security in Cloud Computing Using Cryptography
}

\begin{abstract}
Authors
Chanky Swami ${ }^{1}$, Nishant Anand ${ }^{2}$

${ }^{1}$ M.Tech CSE CBS Group of Institutions, 8 Km. Mile Stone, Jhajjar-Kosli Raod, Fatehpuri, Jhajjar

${ }^{2}$ H.O.D, CSE Dept CBS Group of Institutions, 8 Km.Mile Stone, Jhajjar-Kosli Raod, Fatehpuri, Jhajjar

Email: c.innovator@gmail.com

ABSTRACT

A few malicious persons could use to Cloud computing for unlawful \& criminal \& active use of cloud for illicit intentions is called cloud ill-use. The biggest need is to maintain, manage information resources in digital format \& made resource sharable for multiple access. Information is most vital piece of cloud services so gigantic concern ought to be given to its security. A few malicious persons can utilize Cloud computing for unlawful and criminal and active use of cloud for illicit intentions is known cloud ill-use. The biggest need is to maintain, manage information resources in digital format and made resource sharable for multiple access. Every organization is building its resources either by digitizing documents or by entering new data in digital form. Digital resources available are maintained and are being shared frequently over Cloud Network. Every organization is building its resources either by digitizing documents or by entering new data in digital form. Digital resources available are maintained \& are being shared frequently over Cloud Network.
\end{abstract}

\section{INTRODUCTION}

Security is a standout amongst most concerning issue of Information engineering Issue. To keep client information is essential concern. In event that association's information is not secured on cloud then there is no utilization of moving from old innovation to cloud server. There are parcel of Non-Profit Organization which are helping \& making mindfulness about safety issues of Cloud computing. One of such association is Cloud Security Alliance that given a report in each year in regards to most special security issues in Cloud computing. In 2013 according to CSA reports they distinguished eight famous security dangers to cloud which could mischief client's individual information without knowing them.

Those are as follows:

1. Misuse of Cloud Computing

2. Weak \& insecure Application Software Interface

3. Insider Theft
4. Vulnerabilities in shared cloud services.

5. Data Damage \& loss

6. Data Breaching.

7. Brute Force Attack

8. Service Denial Attack

\section{Video as Multimedia}

Video format consists of different technology concept: one is containers \& another is codec. Container basically describes structure of file: where various pieces are stored, how they are interleaved \& which codec are being used by which pieces. It might specify an audio codec as well as video. It is used to package video \& its components \& is know by a file such as .AVI, .MP4 etc. A codec of audio or video into a stream of bytes such as H.264, MPEG1 etc. It is method used to encode video \& is chief determiner of quality.In past decade, Real time video streaming has become one of most popular applications over Internet. All researchers have been witnessed a 
number of success commercial deployments with CDN or peer to-peer based engines. former achieves high availability \& very short startup latencies, but suffers from excessive costs for deploy dedicated servers. It is particularly severe if user demand fluctuates significantly \& servers have to be over-provisioned for peak loads. peerto-peer solution generally incurs lower deployment cost \& is more scalable, but reliability $\&$ hence service quality could hardly be sure. There have been also efforts toward synergizing dedicated servers with peer to peer. Unfortunately, having an all-round scalable, reliable, responsive, $\&$ cost effectiveness solution remains an elusive goal.

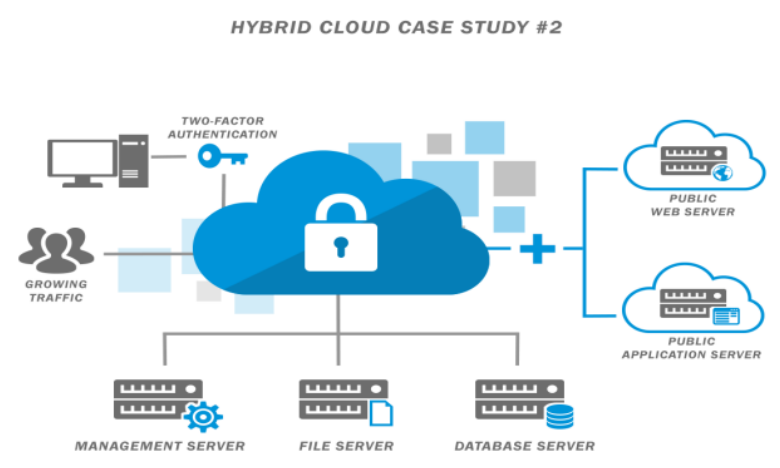

Fig 1 Cloud server Security

\section{LITERATURE REVIEW}

\section{Rahat Afreen \& S.C. Mehrotra (2011)}

This paper deals with cryptography that makes use of public key also called as PKC systems in less. In systems is pertaining to PKC, two keys that are entirely different are utilized for encoding $\&$ decoding data. The strength $\&$ security depends on large key size as among two keys one is distributed \& kept open to public in PKC systems. Previously in PKC systems mathematical problems of prime factorization \& discrete logarithm are used. The security with a almost equal to above with relatively small sizes of key has been promised by ECC \& it has been proved. The ECC are implementation in application specific systems is main point focused in field of research. The requirement of separate crypto coprocessor could be reduced by doing a research in category of ECC mainly related to present many combinations of speed optimized algorithms. Various mathematical techniques are considered to enhance speed \& security of ECC. [116]

\section{Paul Kocher, Ruby Lee, Gary McGraw, Anand Raghunathan \& Srivaths Ravi (2004)}

In this paper security select of embedded has been discussed in detail. A case study that takes into account efforts that is needed for securing electronic systems has been created with increasing cases where data from systems concerned with embedded are being hacked \& destroyed leading to huge loss in last few year. Systems in embedded world that are dedicatedly \& specifically utilized to capture data \& then storing it $\&$ accessing it when required keeping its security sensitivity do possess a large security \& other challenges in providing full security to data. In world of cryptography computing \& security to networks are subject of intensive research. However, inclusion of added features like algorithms that are specific to cryptography \& protocols pertaining to security, to system is often taken by mistake \& misunderstood as security by embedded system designers.

\section{Junfeng Fan, Kazuo Sakiyama \& Ingrid Verbauwhede (2009)}

In this paper they executed Elliptic Curve Cryptography (ECC) on an multi core embedded system, \& methods for scheduling task at different kinds of levels has been studied in detail. A rule for scheduling instructions that makes use of cores to pull off functions in parallel a single modular operation has been proposed. Then, multiple cores performs multiple modular operations in parallel are executed. As method for scheduling joining above two types of parallelism was put forward by doing a comparative study with above two implementations. Here Elliptic curve cryptography's field programmable gate array has been used \& implemented over a prime field. 


\section{TOOLS \& TECHNOLOGY}

Research studies reveal that any authentication mechanism related to web applications \& cloud should provide high security, easy to use interface \& support user mobility. Consumers prefer to access their applications from different locations $\&$ different devices such as desktop, laptop, PDA, smart phones, cell phones etc. Cloud service providers need to ensure that only legitimate users are accessing their services \& this points out to requirement of a strong user authentication mechanism. But there exists various attacks that could create loop holes in verification mechanism $\&$ hence identifying most secure authentication mechanism with high user acceptability is a big challenge in cloud environment. Thus an in-depth idea of attacks on originality \& consistent prevention techniques are required to draft a complete proof authentication mechanism for cloud environment.

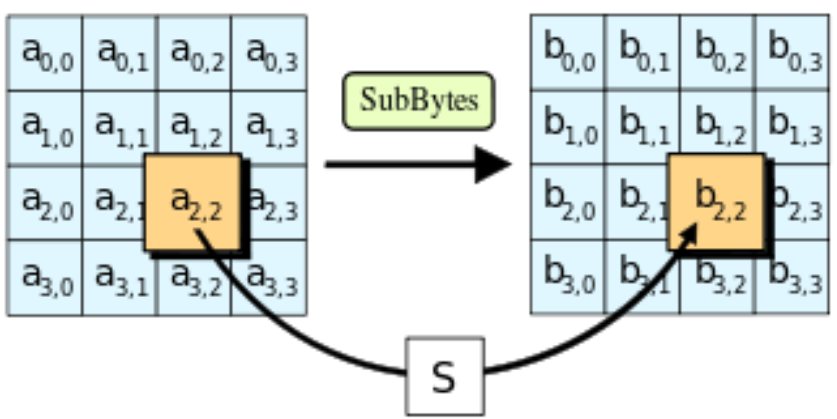

Fig 2 In SubBytes step, each byte in state is replaced with its entry in a fixed 8-bit lookup table, $S ; b_{i j}=S\left(a_{i j}\right)$.

The ShiftRows step

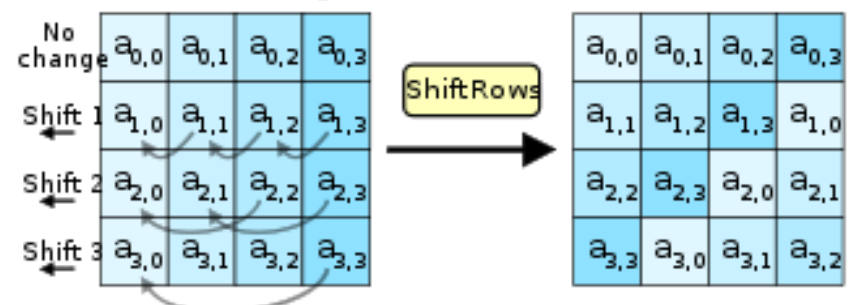

Fig 3 In Shift Rows step, bytes in each row of state are shifted cyclically to left. number of places each byte is shifted differs for each row.

The Mix Columns step

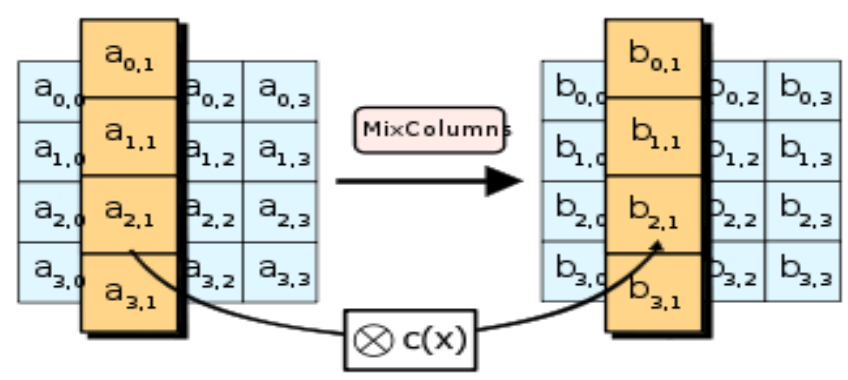

Fig 4 In Mix Columns step, each column of state is multiplied with a fixed polynomial $c(x)$.

\section{PROPOSED IMPLEMENTATION}

Security is becoming an escalating concern in an increasingly multimedia defined world. recent emergence of embedded multimedia applications such as mobile-TV, video messaging, \& telemedicine have increased impact of multimedia $\&$ its security on our personal lives. For example, a significant increase in application of distributed video surveillance technology to monitor traffic \& public places has raised concerns regarding privacy \& security of targeted subjects.

Multimedia content encryption has attracted more \& more researchers \& engineers owing to challenging nature of problem \& its interdisciplinary nature in light of challenges faced with requirements of multimedia communications, multimedia retrieval, multimedia compression \& hardware resource usage.
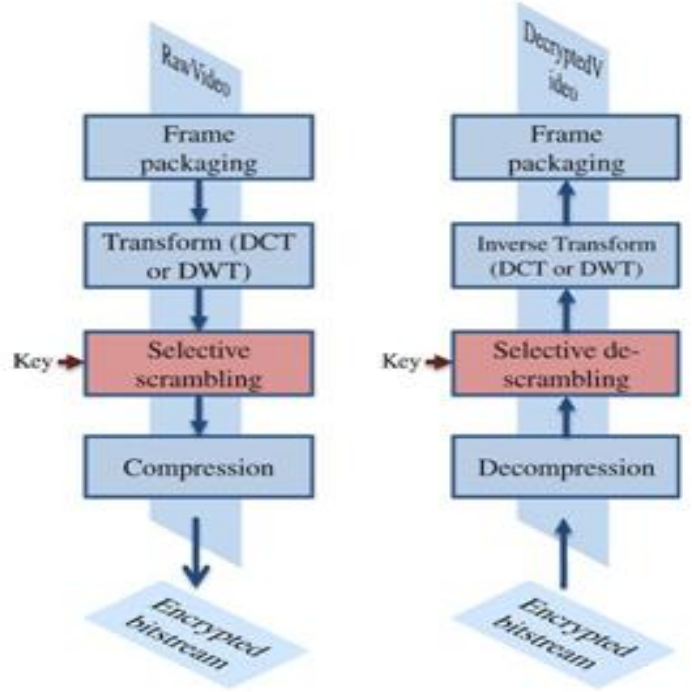

Fig 5 Joint scrambling $\boldsymbol{\&}$ compression framework 


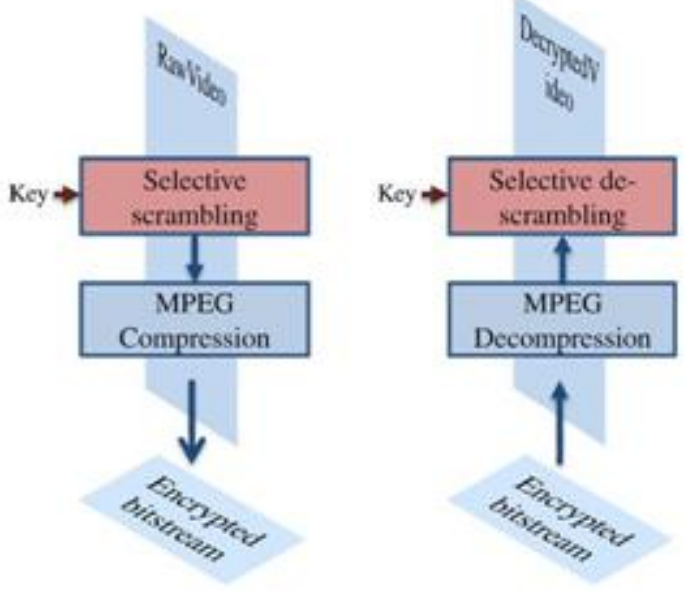

Fig 6 Pre-compression encryption scheme proposed by Pazarci \& Diplin. scrambler allows unauthorized user to have an arbitrarily degraded view of program, yet is totally transparent to MPEG-2 compression

\section{IMPLEMENTATION}

In order to simulate encryption \& decryption of multimedia file we need following:

\section{Hardware Requirements}

- CPU (1GHZ or above)

- $\operatorname{RAM}(1$ GB OR MORE)

- 5GB FREE SPACE IN HARDDISK

- HIGH RESOLUTION MONITOR

\section{Software Requirements}

- Windows $7 / 8$

- JDK(Java Development Kit)

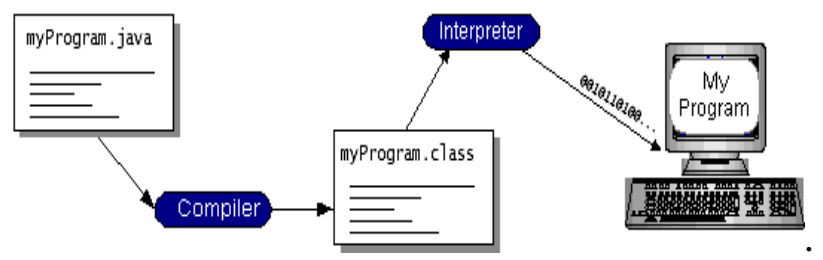

Fig 7 Java Architecture

\section{Socket Programming}

Socket programming is used for communication between applications running on different JRE.

Socket programming could be connectionoriented or connection-less. Socket classes \& Server Socket classes are being used for connection-oriented socket programming \& Data gram Socket \& Packet classes are used for connection-less socket programming.
The client in socket programming must know the below information:

1. Port number, and

2. IP Address of Server

Socket class:

A socket is simply an endpoint for communications between machines. The Socket class could be used to create a socket.

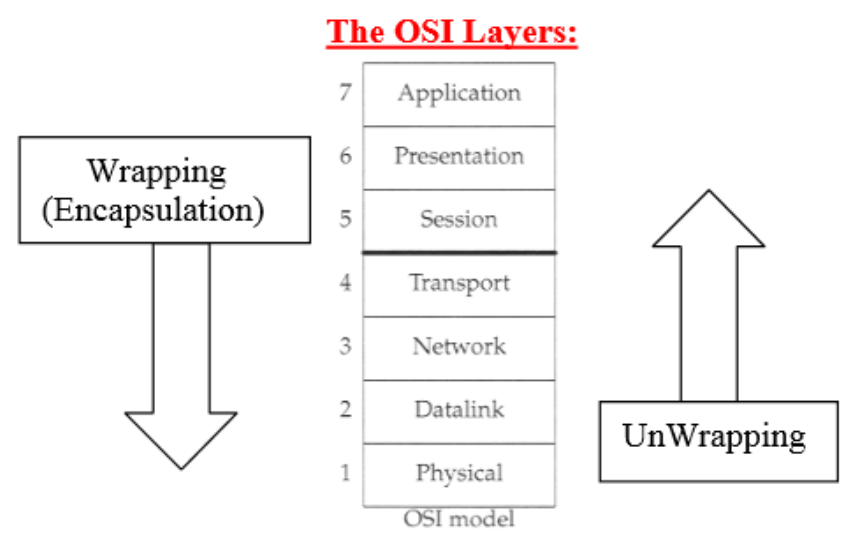

Fig 8 The Socket Interface \& Features of a TCP connection

\section{CONCLUSION}

The main idea behind research is to integrate encryption into compression operation by parameterization of compression blocks, \& (in general) not modifying compressed bits. Two main density blocks where these techniques have been applied are Wavelet Transform \& Entropy Coding.

Encryption into a one operation maked it feasible for cloud servers, mobile \& embedded devices to ensure multimedia security within their less power budgets. By integrating compression \& encryption operations into one these approaches reduce latency of encryption operation which is useful to for real-time video delivery. Our research typically do not change compressed bit streams themselves but change way compressed bitstream is obtained. This integration allows exploiting hierarchical one presentation in a transform domain, as used by most image \& video compression techniques, in order to provide higher functionalities required by more modern applications. 
By giving security for many Multimedia applications like Video on-Demand service, video-conferencing \& multimedia mails is must. A secured video transmission ensures user such that no unapproved eavesdroppers could get information from video while it's being sent to receiver i.e. users those who paid for these services could only watch videos \& movies. If video is more redundant it helps attacker to easily rebuild original video file. Data such as text \& program code has less redundancy as compared to videos in its structure. All these points make providing security for a MPEG video more challenging. Providing security to these MPEG video transmission involves in encrypting parts of MPEG bit stream or entire bit stream.

\section{REFERENCES}

1. Agi, I., Gong, L.: An empirical study of secure mpeg video transmissions. In: Proceedings of Symposium on Network \& Distributed System Security, pp. 137-144. IEEE Press, New York (1996)

2. Baugher, M., McGrew, D., Naslund, M., Carrara, E., Norrman, K.: The secure realtime trans-port protocol (SRTP) (2004)

3. Bergeron, C., Lamy-Bergot, C.: Complaint selective encryption for h.264/avc video streams. In: IEEE 7th Workshop on Multimedia Signal Processing, pp. 1-4 (2005). doi: 10.1109/ MMSP.2005.248641

4. Cheng, H., Li, X.: Partial encryption of compressed images \& videos. IEEE Trans. Signal Process. 48(8), 2439-2451 (2000). doi: $10.1109 / 78.852023$

5. Chiaraluce, F., Ciccarelli, L., Gambi, E., Pierleoni, P., Reginelli, M.: A new chaotic algorithm for video encryption. IEEE Trans. Consum. Electron. 48(4), 838-844 (2002)

6. Li, S., Zheng, X., Mou, X., Cai, Y.: Chaotic encryption scheme for real-time digital video. In: Real-Time Imaging VI.
Proceedings of SPIE, vol. 4666, pp. 149160 (2002)

7. Lian, S., Liu, Z., Ren, Z., Wang, H.: Secure advanced video coding based on selective encryp-tion algorithms. IEEE Trans. Consum. Electron. 52(2), 621-629 (2006)

8. Lian, S., Liu, Z., Ren, Z., Wang, H.: Commutative encryption \& watermarking in video compression. IEEE Trans. Circuits Syst. Video Technol. 17(6), 774778 (2007)

9. Logik Bomb: Hacker's Encyclopedia (1997)

10. Hafner, Katie; Markoff, John (1991). Cyberpunk: Outlaws \& Hackers on Computer Frontier. New York: Simon \& Schuster. ISBN 0-671-68322-5.

11. Sterling, Bruce (1992). The Hacker Crackdown. Bantam. ISBN 0-553-08058$\mathrm{X}$.

12. Slatalla, Michelle; Joshua Quittner (1995). Masters of Deception: The Gang That Ruled Cyberspace. HarperCollins. ISBN 0-06-017030-1.

13. Dreyfus, Suelette (1997). Underground: Tales of Hacking, Madness \& Obsession on Electronic Frontier. Mandarin. ISBN 1-86330-595-5.

14. Verton, Dan (2002). The Hacker Diaries : Confessions of Teenage Hackers. McGraw-Hill Osborne Media. ISBN 0-07222364-2.

15. Thomas, Douglas (2002). Hacker Culture. University of Minnesota Press. ISBN 08166-3345-2.

16. Taylor, Paul A. (1999). Hackers: Crime in Digital Sublime. Routledge. ISBN 978-0415-18072-

17. Levy, Steven (2002). Crypto: How Code Rebels Beat Government Saving Privacy in Digital Age. Penguin. ISBN 0-14024432-8. 
18. Ventre, Daniel (2009). Information Warfare. Wiley - ISTE. ISBN 978-184821-094-3.

19. Bhushan Lal Sahu,Rajesh Tiwari, Journal of Advanced Research in Computer Science \& Software Engineering 2(9) (2012) 33-37.

20. Peter Mell, Tim Grance (2011). The NIST Definition of Cloud Computing, National Institute of Standards \& Technology Report. 2011.

21. Sultan Ullah, Zheng Xuefeng (2013). Cloud Computing Research Challenges. IEEE $5^{\text {th }}$ International Conference on Biomedical Engineering \& Informatics, pp 1397-1401.

22. Tripathi A., Mishra A. (2011). Cloud Computing Security Considerations. Signal Processing, Communications \& Computing (ICSPCC), IEEE International Conference.

23. Mohammad Reza Modarres Zadeh, International Letters of Social \& Humanistic Sciences 3 (2013) 21-29.

24. Leah Garner-O’Neale, Jelisa Maughan, Babalola Ogunkola, International Letters of Social \& Humanistic Sciences 2 (2014) 41-55.

25. Gaines, Helen Fouché (1939). Cryptanalysis, Dover, ISBN 0-486-200973. Considered one of classic books on subject, \& includes many sample ciphertext for practice. It reflects public amateur practice as of inter-War period. The book was compiled as one of first projects of American Cryptogram Association. 